\title{
Solution of the Poisson equation for infinite and semi-infinite systems including near field corrections for charge densities of arbitrary shape
}

\author{
R. HAMMERLING $\dagger$, J. ZABLOUDIL $\dagger$, L. SZUNYOGH $\dagger+$ \\ and P. WEINBERGER*† \\ $\dagger$ Center for Computational Material Science, Vienna University of Technology, \\ Getreidemarkt 9/134, A-1060 Vienna, Austria \\ tDepartment of Theoretical Physics and Center for Applied Mathematics and \\ Computational Physics, Budapest University of Technology and Economics, \\ Budafoki út 8, H-1521 Budapest, Hungary
}

(Received 29 April 2005; in final form 3 August 2005)

\begin{abstract}
A method for solving the Poisson equation is presented for systems with three- or two-dimensional translational lattice symmetry, the latter applying to surfaces, interfaces or slabs. Special attention is given to the so-called near field correction (NFC), namely to a correction to the electrostatic potential arising from neighbouring (or near) cells, that is inherent, e.g. to the full-potential KKR method. The results of numerical tests presented serve to illustrate the effect of the NFC. Furthermore, the question of the convergence of 'internal' angular momentum sums is addressed and discussed in detail.
\end{abstract}

\section{Introduction}

The Screened Korringa-Kohn-Rostoker method (SKKR) which was developed about ten years ago [1] has been successfully applied in the past to a large number of problems. This method is especially suitable for systems with a two-dimensional lattice translational symmetry such as surfaces or interfaces but can also be used to calculate bulk properties. In order to extend the SKKR method to a full potential description, by partitioning the configuration space into non-overlapping but spacefilling cells to which the individual potentials and the charge densities are confined, an appropriate method for solving Poisson's equation needs to be developed.

Within multiple scattering theory [2] the charge density is evaluated in terms of the Green's function of the system and the electrostatic part of the potential subsequently by solving Poisson's equation. According to the requirements of density functional theory this procedure has to be repeated until convergence of the potential is reached. Since it is an underlying feature of multiple scattering methods to expand quantities like the charge density and the potential at cell centres located

*Corresponding author. Email: pw@cms.tuwien.ac.at 
at positions $\mathbf{R}$ into a series of (complex) spherical harmonics, a solution of Poisson's equation consequently also has to be based on a corresponding expansion of the $1 /\left|\mathbf{R}+\mathbf{r}-\mathbf{R}^{\prime}-\mathbf{r}^{\prime}\right|$-like terms, which, however, do not converge for neighbouring sites $\mathbf{R}$ and $\mathbf{R}^{\prime}$ at certain points inside a given cell.

This so-called 'near cell' problem has already been the subject of several publications. Gonis et al. [3] developed a method (later applied by Vitos et al. [4]) based on shifting (and back-shifting) the neighbouring cells with a displacement vector b. Although, in principle, this method should work it suffers from the fact that it results in a conditionally convergent double angular momentum sum from which the convergence of the internal sum seems to be rather slow.

Another method was presented by Zhang et al. [5] in which the internal angular momentum summations are replaced by surface integrals, which, however, may turn out to be quite tedious to calculate for more complex geometries. Furthermore, there are still open questions concerning the fact that the system of linear equations is degenerate (reflecting that an arbitrary constant can be added to the electrostatic potential). The recipe provided in [5], therefore, turns out not to work for certain combinations of multi-atom unit cells and cell shapes. A further method to solve the electrostatic problem was recently proposed in [6]. It is conceptually easy and computationally very effective, however, it might contain numerical convergence problems if used with the characteristic shape functions of a cell. A more detailed comparison of this method and the one proposed in this paper will be presented later on.

The method presented in this paper is based on the idea that the electrostatic potential in one specified cell is the sum of an intracell potential, which is the contribution from the charge density within the chosen cell and an intercell potential which results from the charge distributions in all other cells of the system:

$$
V_{\mathbf{R}}^{\mathrm{el}}(\mathbf{r})=V_{\mathbf{R}}^{\text {Inter }}(\mathbf{r})+V_{\mathbf{R}}^{\text {Intra }}(\mathbf{r}), \quad \mathbf{r} \in \Omega_{\mathbf{R}},
$$

where $\Omega_{\mathbf{R}}$ denotes the Wigner-Seitz cell around lattice site $\mathbf{R}$. While obtaining the intracell potential is straightforward, a calculation of the intercell part is more complicated. In the following for systems with three-dimensional periodic boundary conditions Ewald's method is applied, while in the case of only two-dimensional translational symmetry we followed a derivation similar to that explored by Kambe [7] for the calculation of the structure constants used in LEED theory. In both cases a summation with respect to real and reciprocal lattice vectors is used which leads to rapidly converging series. If the potential is calculated in this way one assumes that the following geometric conditions

$$
r<\left|\mathbf{r}^{\prime}-\mathbf{R}+\mathbf{R}^{\prime}\right| \quad \text { and } \quad r^{\prime}<\left|\mathbf{R}-\mathbf{R}^{\prime}\right|
$$

apply, which of course are not fulfilled by the so-called near cells. Therefore, incorrect contributions arise from neighbouring cells which have to be corrected for. This can be achieved precisely by noting that the intercell potential of a given cell $\mathbf{R}$ is the sum of intracell potentials, centred at their respective origins, over all other cells $\mathbf{R}^{\prime}$,

$$
V_{\mathbf{R}}^{\text {Inter }}(\mathbf{r})=\sum_{\mathbf{R}^{\prime} \neq \mathbf{R}} V_{\mathbf{R}^{\prime}}^{\text {Intra }}\left(\mathbf{R}-\mathbf{R}^{\prime}+\mathbf{r}\right)
$$


By summing up only the contributions of the near cells, while at the same time subtracting the incorrect ones calculated by the combined real and reciprocal space methods (Ewald or Kambe), the true intercell potential can be obtained. These corrected contributions to the intercell potential will be referred to as near field corrections throughout this work.

The paper is structured in the following way: section 2 deals in a very condensed manner with the general solution of Poisson's equation for systems with two- or three-dimensional translational symmetry neglecting the near field corrections (a more detailed derivation can be found in [2] and [8]). In section 3 a method for the calculation of the near field corrections is discussed while section 4 is concerned with numerical tests of the method presented here.

\section{The Poisson equation and the generalized Madelung problem for two- and three-dimensional translationally invariant systems}

Using atomic Rydberg units, in particular, $e^{2}=2$, the Poisson equation is given by

$$
\Delta V(\mathbf{r})=-8 \pi \rho(\mathbf{r}),
$$

and the corresponding Green's function by

$$
G_{0}\left(\mathbf{r}, \mathbf{r}^{\prime}\right)=\frac{1}{\left|\mathbf{r}-\mathbf{r}^{\prime}\right|}, \quad \Delta G_{0}\left(\mathbf{r}, \mathbf{r}^{\prime}\right)=-4 \pi \delta\left(\mathbf{r}-\mathbf{r}^{\prime}\right)
$$

such that

$$
V(\mathbf{r})=2 \int \mathrm{d} \mathbf{r}^{\prime} G\left(\mathbf{r}, \mathbf{r}^{\prime}\right) \rho\left(\mathbf{r}^{\prime}\right)=2 \int \mathrm{d} \mathbf{r}^{\prime} \frac{\rho\left(\mathbf{r}^{\prime}\right)}{\left|\mathbf{r}-\mathbf{r}^{\prime}\right|}
$$

At a particular lattice site $\mathbf{R}$ the intercell contribution to the electrostatic potential is then given by

$$
V_{\mathbf{R}}^{\text {Inter }}(\mathbf{r})=2 \sum_{\substack{\mathbf{R}^{\prime} \\\left(\mathbf{R}^{\prime} \neq \mathbf{R}\right)}} \int_{\Omega_{\mathbf{R}^{\prime}}} \mathrm{d} \mathbf{r}^{\prime} G_{0}\left(\mathbf{r}+\mathbf{R}, \mathbf{r}^{\prime}+\mathbf{R}^{\prime}\right) \rho_{\mathbf{R}^{\prime}}\left(\mathbf{r}^{\prime}\right) ; \quad \mathbf{r} \in \Omega_{\mathbf{R}},
$$

and the intracell contribution to the electrostatic potential by

$$
V_{\mathbf{R}}^{\text {Intra }}(\mathbf{r})=2 \int_{\Omega_{\mathbf{R}}} \mathrm{d} \mathbf{r}^{\prime} G_{0}\left(\mathbf{r}, \mathbf{r}^{\prime}\right) \rho_{\mathbf{R}}\left(\mathbf{r}^{\prime}\right) ; \quad \mathbf{r} \in \Omega_{\mathbf{R}}
$$

\subsection{Intracell contribution}

Let $\bar{\rho}(\mathbf{r})$ be the (shape truncated) charge density in the cell of a chosen origin $\mathbf{R}_{0}$ in an arbitrary ensemble of scatterers,

$$
\bar{\rho}(\mathbf{r})=\rho(\mathbf{r}) \sigma_{\Omega_{0}}(\mathbf{r})=\sum_{L} \bar{\rho}_{L}(r) Y_{L}^{*}(\hat{\mathbf{r}}),
$$


where the index $\Omega_{0}=\Omega_{\mathbf{R}_{0}}$ refers to the domain around $\mathbf{R}_{0}, L$ is a combined orbital angular momentum index $(\ell, m)$ and the $Y_{L}(\hat{\mathbf{r}})$ are (complex) spherical harmonics [9]. The function $\sigma_{\Omega_{0}}(\mathbf{r})$ is called the characteristic or shape function of cell $\Omega_{\mathbf{R}_{0}}$, the calculation of the expansion coefficients $\sigma_{L}(r)$ of which is extensively documented in the literature [10-12]. By using the well-known angular momentum expansion for $1 /\left|\mathbf{r}-\mathbf{r}^{\prime}\right|$, the potential due to the charge distribution inside a particular cell,

$$
V^{\text {Intra }}(\mathbf{r})=2 \int_{\Omega_{0}} \frac{1}{\left|\mathbf{r}-\mathbf{r}^{\prime}\right|} \bar{\rho}\left(\mathbf{r}^{\prime}\right) \mathrm{d} \mathbf{r}^{\prime}
$$

can be written as

$$
V^{\text {Intra }}(\mathbf{r})=\sum_{L} \frac{8 \pi}{2 \ell+1}\left[r^{\ell} \int_{r}^{r_{\mathrm{BS}}} \frac{\bar{\rho}_{L}\left(r^{\prime}\right)}{\left(r^{\prime}\right)^{\ell-1}} \mathrm{~d} r^{\prime}+\frac{1}{r^{\ell+1}} \int_{0}^{r}\left(r^{\prime}\right)^{\ell+2} \bar{\rho}_{L}\left(r^{\prime}\right) \mathrm{d} r^{\prime}\right] Y_{L}^{*}(\hat{\mathbf{r}}) .
$$

The coefficients of the shape truncated charge density $\bar{\rho}_{L}(r)$ are given explicitly by

$$
\bar{\rho}_{L}(r)=\sum_{L^{\prime} L^{\prime \prime}} C_{L^{\prime \prime} L}^{L^{\prime}} \rho_{L^{\prime}}(r) \sigma_{L^{\prime \prime}}(r)
$$

where the $C_{L^{\prime \prime} L}^{L^{\prime}}$ are the well-known Gaunt coefficients. The expansion coefficients of the intracell potential can therefore be written as

$$
\begin{aligned}
V_{L}^{\text {Intra }}(r)= & \frac{8 \pi}{2 \ell+1}\left[r^{\ell} \int_{r}^{r_{\mathrm{BS}}} \frac{1}{\left(r^{\prime}\right)^{\ell-1}}\left(\sum_{L^{\prime} L^{\prime \prime}} C_{L^{\prime \prime} L}^{L^{\prime}} \rho_{L^{\prime}}\left(r^{\prime}\right) \sigma_{L^{\prime \prime}}\left(r^{\prime}\right)\right) \mathrm{d} r^{\prime}\right. \\
& \left.+\frac{1}{r^{\ell+1}} \int_{0}^{r}\left(r^{\prime}\right)^{\ell+2}\left(\sum_{L^{\prime} L^{\prime \prime}} C_{L^{\prime \prime} L}^{L^{\prime}} \rho_{L^{\prime}}\left(r^{\prime}\right) \sigma_{L^{\prime \prime}}\left(r^{\prime}\right)\right) \mathrm{d} r^{\prime}\right]^{*} .
\end{aligned}
$$

\subsection{Multipole expansion in real-space}

Clearly the total charge density is the sum over all local densities $\bar{\rho}_{\mathbf{R}}(\mathbf{r})$ centred at positions $\mathbf{R}$,

$$
\rho(\mathbf{r})=\sum_{\mathbf{R}} \bar{\rho}_{\mathbf{R}}(\mathbf{r}-\mathbf{R}), \quad \bar{\rho}_{\mathbf{R}}(\mathbf{r}-\mathbf{R})=\rho(\mathbf{r}) \sigma_{\Omega_{\mathbf{R}}}(\mathbf{r}-\mathbf{R})
$$

which in turn can be expanded as

$$
\bar{\rho}_{\mathbf{R}}(\mathbf{r})=\sum_{L} \bar{\rho}_{\mathbf{R}, L}(r) Y_{L}^{*}(\hat{\mathbf{r}})
$$

The corresponding spherical multipole moments are then defined by

$$
Q_{\mathbf{R}}^{L}=\frac{(4 \pi)^{1 / 2}}{2 \ell+1} \int_{\Omega_{\mathbf{R}}} r^{\ell} \bar{\rho}_{\mathbf{R}}(\mathbf{r}) Y_{L}(\hat{\mathbf{r}}) \mathrm{d} \mathbf{r}
$$


or, expressed in terms of the expansion coefficients of the untruncated charge density, $\rho_{\mathbf{R}, L}(r)$, by

$$
Q_{\mathbf{R}}^{L}=\frac{(4 \pi)^{1 / 2}}{2 \ell+1} \sum_{L^{\prime} L^{\prime \prime}} C_{L^{\prime \prime} L}^{L^{\prime}} \int_{0}^{r_{\mathrm{BS}}} r^{\ell+2} \rho_{\mathbf{R}, L^{\prime}}(r) \sigma_{\mathbf{R}, L^{\prime \prime}}(r) \mathrm{d} r
$$

\subsection{Green's functions and Madelung constants}

For

$$
r<\left|\mathbf{r}^{\prime}-\mathbf{R}+\mathbf{R}^{\prime}\right| \quad \text { and } \quad r^{\prime}<\left|\mathbf{R}-\mathbf{R}^{\prime}\right|
$$

the Green's function,

$$
G_{0}\left(\mathbf{r}+\mathbf{R}, \mathbf{r}^{\prime}+\mathbf{R}^{\prime}\right)=\frac{1}{\left|\mathbf{r}+\mathbf{R}-\mathbf{r}^{\prime}-\mathbf{R}^{\prime}\right|},
$$

can be reformulated by using angular momentum expansions for $1 /\left|\mathbf{r}-\mathbf{r}^{\prime}\right|$ as

$$
G_{0}\left(\mathbf{r}+\mathbf{R}, \mathbf{r}^{\prime}+\mathbf{R}^{\prime}\right)=\sum_{L L^{\prime}} \frac{(4 \pi)^{1 / 2}}{2 \ell+1} r^{\ell} Y_{L}^{*}(\widehat{\mathbf{r}}) A_{\mathbf{R R}^{\prime}}^{L L^{\prime}} \frac{(4 \pi)^{1 / 2}}{2 \ell^{\prime}+1}\left(r^{\prime}\right)^{\ell^{\prime}} Y_{L^{\prime}}\left(\widehat{\mathbf{r}}^{\prime}\right),
$$

where the matrix elements $A_{\mathbf{R R}^{\prime}}^{L L^{\prime}}$,

$$
A_{\mathbf{R}^{\prime}}^{L L^{\prime}}=(-1)^{\ell} \frac{4 \pi\left[2\left(\ell+\ell^{\prime}\right)-1\right] ! !}{(2 \ell-1) ! !\left(2 \ell^{\prime}-1\right) ! !} C_{\ell m,\left(\ell+\ell^{\prime}\right)\left(m^{\prime}-m\right)}^{\ell^{\prime} m^{\prime}} \frac{Y_{\left(\ell+\ell^{\prime}\right)\left(m^{\prime}-m\right)}^{*}\left(\widehat{\mathbf{R}-\mathbf{R}^{\prime}}\right)}{\left|\mathbf{R}-\mathbf{R}^{\prime}\right|^{\ell+\ell^{\prime}+1}},
$$

are usually called the real-space Madelung constants for two centres. The assumption (18) implies that the bounding spheres of the cells at $\mathbf{R}$ and $\mathbf{R}^{\prime}$ must not overlap. By neglecting near field corrections, to which section 3 is devoted, the intercell potential in (7) can then be expressed as

$$
\begin{aligned}
V_{\mathbf{R}}^{\text {Inter }}(\mathbf{r}) & =2 \sum_{\mathbf{R}^{\prime}(\neq \mathbf{R})} \sum_{L L^{\prime}} \frac{(4 \pi)^{1 / 2}}{2 \ell+1} r^{\ell} Y_{L}^{*}(\widehat{\mathbf{r}}) A_{\mathbf{R}^{\prime}}^{L L^{\prime}} Q_{\mathbf{R}^{\prime}}^{L^{\prime}} \\
& =\sum_{L} V_{\mathbf{R}, L}^{\mathrm{Inter}}(r)^{*} Y_{L}^{*}(\widehat{\mathbf{r}})=\sum_{L} V_{\mathbf{R}, L}^{\mathrm{Inter}}(r) Y_{L}(\widehat{\mathbf{r}})
\end{aligned}
$$

where

$$
V_{\mathbf{R}, L}^{\mathrm{Inter}}(r)=\frac{4 \pi^{1 / 2}}{2 \ell+1}\left(\sum_{\mathbf{R}^{\prime}(\neq \mathbf{R})} \sum_{L^{\prime}} A_{\mathbf{R}^{\prime}}^{L L^{\prime}} Q_{\mathbf{R}^{\prime}}^{L^{\prime}}\right)^{*} r^{\ell}
$$


Evidently equation (21) can be reformulated as $\dagger$

$$
\begin{aligned}
A_{\mathbf{R R}^{\prime}}^{L L^{\prime}} & =(-1)^{\ell^{\prime}} \frac{\left[2\left(\ell+\ell^{\prime}\right)+1\right] ! !}{(2 \ell-1) ! !\left(2 \ell^{\prime}-1\right) ! !} C_{\ell m,\left(\ell+\ell^{\prime}\right)\left(m^{\prime}-m\right)}^{\ell^{\prime} m^{\prime}} G_{\mathbf{R R}^{\prime}}^{\left(\ell+\ell^{\prime}\right)\left(m^{\prime}-m\right)} \\
& =2 \pi^{1 / 2}(-1)^{\ell^{\prime}} \frac{\Gamma\left(\ell+\ell^{\prime}+\frac{3}{2}\right)}{\Gamma\left(\ell+\frac{1}{2}\right) \Gamma\left(\ell^{\prime}+\frac{1}{2}\right)} C_{\ell m,\left(\ell+\ell^{\prime}\right)\left(m^{\prime}-m\right)}^{\ell^{\prime} m^{\prime}} G_{\mathbf{R R}^{\prime}}^{\left(\ell+\ell^{\prime}\right)\left(m^{\prime}-m\right)},
\end{aligned}
$$

with

$$
G_{\mathbf{R}^{\prime}}^{L}=\frac{4 \pi}{2 \ell+1} \frac{Y_{L}^{*}\left(\widehat{\mathbf{R}^{\prime}-\mathbf{R}}\right)}{\left|\mathbf{R}-\mathbf{R}^{\prime}\right|^{\ell+1}}=\frac{4 \pi(-1)^{\ell}}{2 \ell+1} \frac{Y_{L}^{*}\left(\widehat{\mathbf{R}-\mathbf{R}^{\prime}}\right)}{\left|\mathbf{R}-\mathbf{R}^{\prime}\right|^{\ell+1}} .
$$

In principle the coefficients $V_{\mathbf{R}, L}^{\mathrm{Inter}}(r)$ can be evaluated by means of the direct space summation in (23), which (if at all) leads to a slowly converging series. In the following therefore use of the underlying two- or three-dimensional translational invariance is made. Because of the relationship in equation (24), in the following all expressions will be formulated in terms of reduced Madelung constants $G_{\mathbf{R}^{\prime}}^{L}$.

\subsection{Three-dimensional complex lattices}

Let $\mathbf{R}_{n \mu}$ denote the positions in a (in general) complex three-dimensional lattice $\left(\mathcal{L}^{(3)}\right)$

$$
\mathbf{R}_{n \mu}=\mathbf{t}_{n}+\mathbf{a}_{\mu},
$$

where the $\mathbf{t}_{n} \in \mathcal{L}^{(3)}$ are lattice translations, the $\mathbf{a}_{\mu}$ refer to inequivalent atomic positions and $\mathcal{L}^{(3)}$ denotes the $3 \mathrm{D}$ translational lattice. The total electrostatic potential then obviously depends only on the 'sublattice' index $\mu$, i.e. is independent of $n$,

$$
\begin{aligned}
V\left(\mathbf{R}_{n \mu}+\mathbf{r}\right) & =V\left(\mathbf{a}_{\mu}+\mathbf{r}\right)=V_{\mu}(\mathbf{r}) \\
& =2 \sum_{n, v} \int_{\Omega_{v}} \mathrm{~d} \mathbf{r}^{\prime} G_{0}\left(\mathbf{a}_{\mu}+\mathbf{r}, \mathbf{a}_{v}+\mathbf{t}_{n}+\mathbf{r}^{\prime}\right) \rho_{\nu}\left(\mathbf{r}^{\prime}\right), \quad \mathbf{r} \in \Omega_{\mu},
\end{aligned}
$$

where

$$
\rho_{\mu}(\mathbf{r})=\rho_{\mathbf{R}_{n \mu}}(\mathbf{r})
$$

In this case $\Omega$ picks up the meaning of a periodically repeated cell in sublattice $\mu$.

In order to separate those parts in (27) that are independent of the charge density, one first performs a summation over all $n$, i.e. over all $\mathbf{t}_{n} \in \mathcal{L}^{(3)}$,

$$
G_{\mu \nu}\left(\mathbf{r}, \mathbf{r}^{\prime}\right)=\sum_{\substack{\mathbf{t}_{n} \\\left(\mu=\nu, \mathbf{t}_{n} \neq 0\right)}} G_{0}\left(\mathbf{r}+\mathbf{a}_{\mu}, \mathbf{r}^{\prime}+\mathbf{t}_{n}+\mathbf{a}_{v}\right),
$$

$\uparrow$ All standard functions used are defined in [13] and [14]. 
and only then sums over all sublattices

$$
V_{\mu}^{\mathrm{Inter}}(\mathbf{r})=2 \sum_{\nu} \int_{\Omega_{\nu}} \mathrm{d} \mathbf{r}^{\prime} G_{\mu \nu}\left(\mathbf{r}, \mathbf{r}^{\prime}\right) \rho_{\nu}\left(\mathbf{r}^{\prime}\right), \quad \mathbf{r} \in \Omega_{\mu}
$$

Applying the Ewald technique by combining real and reciprocal summations leads to an absolute convergent series (with $\mathbf{a}_{\mu \nu}=\mathbf{a}_{\mu}-\mathbf{a}_{v}$ ):

$$
\begin{aligned}
G_{\mu \nu}(\mathbf{r})= & \frac{4 \pi}{V} \sum_{\mathbf{g}_{j} \neq \mathbf{0}} \exp \left(\mathbf{i g}_{j} \mathbf{r}\right) \exp \left(\mathbf{i g}_{j} \mathbf{a}_{\mu \nu}\right) \frac{\exp \left(-\mathbf{g}_{j}^{2} \sigma^{2}\right)}{\mathbf{g}_{j}^{2}} \\
& +\sum_{\substack{\mathbf{t}_{n} \neq \\
\left(\mu=t_{n} \neq \mathbf{0}\right)}} \frac{1}{\left|\mathbf{r}+\mathbf{a}_{\mu \nu}-\mathbf{t}_{n}\right|} \operatorname{erfc}\left(\left|\mathbf{r}+\mathbf{a}_{\mu \nu}-\mathbf{t}_{n}\right| / 2 \sigma\right) \\
& -\delta_{\mu \nu} \frac{\operatorname{erf}(|\mathbf{r}| / 2 \sigma)}{|\mathbf{r}|}-\frac{4 \pi \sigma^{2}}{V},
\end{aligned}
$$

where $\sigma$ is the Ewald parameter and the coefficients of the expansion,

$$
G_{\mu \nu}(\mathbf{r})=\sum_{L} G_{\mu \nu}^{L}|\mathbf{r}|^{\ell} Y_{L}(\hat{\mathbf{r}})
$$

are given by

$$
\begin{aligned}
G_{\mu \nu}^{L}(\mathbf{r})= & \frac{(4 \pi)^{2} \pi^{1 / 2} i^{\ell}}{V 2^{\ell+1} \Gamma\left(\ell+\frac{3}{2}\right)} \sum_{\mathbf{g}_{j} \neq \mathbf{0}} Y_{L}^{*}\left(\hat{\mathbf{g}}_{j}\right) \exp \left(\mathbf{i g}_{j} \mathbf{a}_{\mu \nu}\right)\left|\mathbf{g}_{j}\right|^{\ell-2} \exp \left(-\mathbf{g}_{j}^{2} \sigma^{2}\right) \\
& +\frac{2 \pi(-1)^{\ell}}{\Gamma\left(\ell+\frac{3}{2}\right)} \sum_{\substack{\mathbf{t}_{n} \\
\left(\mu \nu \mathbf{v}_{n} \neq 0\right)}} Y_{L}^{*}\left(\mathbf{a}_{\mu \nu}-\mathbf{t}_{n}\right) \frac{\Gamma\left(\ell+(1 / 2),\left|\mathbf{a}_{\mu \nu}-\mathbf{t}_{n}\right|^{2} / 4 \sigma^{2}\right)}{\left|\mathbf{a}_{\mu \nu}-\mathbf{t}_{n}\right|^{\ell+1}} \\
& -\delta_{L,(0,0)}\left(\frac{(4 \pi)^{3 / 2} \sigma^{2}}{V}+\delta_{\mu \nu} \frac{2}{\sigma}\right) .
\end{aligned}
$$

In terms of these quantities the potential can now be written as

$$
V_{\mu}^{\mathrm{Inter}}(\mathbf{r})=\sum_{L} V_{\mu, L}^{\mathrm{Inter}}(r) Y_{L}(\hat{\mathbf{r}})
$$

with coefficients $V_{\mu, L}^{\mathrm{Inter}}(r)=V_{\mu, \ell m}^{\mathrm{Inter}}(r)$,

$$
\begin{aligned}
& V_{\mu, \ell m}^{\mathrm{Inter}}(r) \\
& \quad=\frac{4 \pi}{\Gamma\left(\ell+\frac{3}{2}\right)}\left(\sum_{L^{\prime}} \frac{(-1)^{\ell^{\prime}} \Gamma\left(\ell+\ell^{\prime}+\frac{3}{2}\right)}{\Gamma\left(\ell^{\prime}+\frac{1}{2}\right)} C_{\ell m,\left(\ell+\ell^{\prime}\right)\left(m^{\prime}-m\right)}^{\ell^{\prime} m^{\prime}} \sum_{\nu} G_{\mu \nu}^{\ell+\ell^{\prime}, m^{\prime}-m} Q_{\nu}^{L^{\prime}}\right)^{*} r^{\ell} .
\end{aligned}
$$


It should be recalled that one only needs to evaluate $V_{\mu, \ell m}^{\text {Inter }}(r)$ for $m \geq 0$, since the intercell potential $V_{\mu}^{\text {Inter }}(\mathbf{r})$ is real.

\subsection{Complex two-dimensional lattices}

In the case of two-dimensional translational invariance $\left(\mathcal{L}^{(2)}\right)$ all atomic positions can be written as

$$
\mathbf{R}_{n p}=\mathbf{t}_{n}+\mathbf{c}_{p},
$$

where $\mathbf{t}_{n} \in \mathcal{L}^{(2)}$ is a lattice translation and $\mathbf{c}_{p}$ are inequivalent atomic positions, also called layer position vectors. As before the charge density is independent of lattice translations,

$$
\rho_{\mathbf{R}_{n p}}(\mathbf{r})=\rho_{p}(\mathbf{r}), \quad \forall \mathbf{t}_{n} \in \mathcal{L}^{(2)}
$$

and, therefore, the potential depends again only on the layer index $p$,

$$
\begin{aligned}
V_{p}(\mathbf{r}) & =V\left(\mathbf{c}_{p}+\mathbf{r}\right)=2 \sum_{q} \int_{\Omega_{q}} \mathrm{~d} \mathbf{r}^{\prime} G_{p q}\left(\mathbf{r}, \mathbf{r}^{\prime}\right) \rho_{q}\left(\mathbf{r}^{\prime}\right), \quad \mathbf{r} \in \Omega_{p} \\
G_{p q}\left(\mathbf{r}, \mathbf{r}^{\prime}\right) & =G_{p q}\left(\mathbf{r}-\mathbf{r}^{\prime}\right)=\sum_{\substack{\mathbf{t}_{n} \\
\left(p=q, \mathbf{t}_{n} \neq 0\right)}} G_{0}\left(\mathbf{r}+\mathbf{c}_{p}, \mathbf{r}^{\prime}+\mathbf{t}_{n}+\mathbf{c}_{q}\right) .
\end{aligned}
$$

A method similar to the one of Kambe for the LEED structure constants [7] leads to an expression for $G_{p q}\left(\mathbf{r}-\mathbf{r}^{\prime}\right)$ and the expansion,

$$
G_{p q}(\mathbf{r})=\sum_{L} G_{p q}^{L}|\mathbf{r}|^{\ell} Y_{L}(\hat{\mathbf{r}})
$$

yields the reduced Madelung structure constants $G_{p q}^{L}$ for a 2D lattice [8]. They are given by the following expressions

$$
G_{p q}^{L}=\sum_{i=0,1,2 \mathrm{a}, 2 \mathrm{~b}, 3} D_{i, p q}^{L}=D_{0, p q}^{L}+D_{1, p q}^{L}+D_{2 a, p q}^{L}+D_{2 b, p q}^{L}+D_{3, p q}^{L}
$$

for the various contributions $D_{i, p q}^{L}$ :

$$
D_{0, p q}^{L}=-\left(1-\delta_{c_{p q \perp}, 0}\right) \delta_{m 0} \frac{4 \pi}{A}\left(\delta_{\ell 0} \pi^{1 / 2}\left|c_{p q \perp}\right|+\delta_{\ell 1} \frac{(3 \pi)^{1 / 2}}{3} \operatorname{sign}\left(c_{p q \perp}\right)\right)
$$

where $A$ is the area of the $2 \mathrm{D}$ unit cell and $c_{p q}=c_{p}-c_{q}$. 
With reciprocal lattice vectors $\mathbf{g}_{j}=\left(g_{j} \cos \left(\phi_{j}\right), g_{j} \sin \left(\phi_{j}\right)\right)$ the reciprocal sum contribution can be written as

$$
\begin{aligned}
D_{1, p q}^{L}= & \frac{\pi^{3 / 2} \mathrm{i}^{-m}}{2^{2 \ell-1} A} \frac{[(2 \ell+1) \Gamma(\ell+|m|+1) \Gamma(\ell-|m|+1)]^{1 / 2}}{\Gamma\left(\ell+\frac{3}{2}\right)} \\
& \times \sum_{\substack{\mathbf{g}_{j} \\
\left(\mathbf{g}_{j} \neq 0\right)}}\left\{\exp \left(-\mathrm{i} m \phi_{j}\right) \exp \left(\mathbf{i g}_{j} \cdot \mathbf{c}_{p q \|}\right)\right. \\
& \times \sum_{k=0}^{\ell-|m| / 2} \sum_{n=(\ell-|m| / 2)-k}^{\ell-|m|-2 k} I_{n}\left(g_{j} \sigma, \frac{\left|c_{p q \perp}\right| g_{j}}{2}\right) \\
& \times \frac{(-1)^{n} c_{p q \perp}^{2 n-\ell+|m|+2 k}}{\Gamma(2 n-\ell+|m|+2 k+1) \Gamma(\ell-|m|-n-2 k+1)} \\
& \left.\times \frac{g_{j}^{2 n+|m|+2 k-1}}{\Gamma(k+1) \Gamma(|m|+k+1)}\right\}
\end{aligned}
$$

where the occurring integral

$$
I_{n}\left(g_{j} \sigma, \frac{\left|c_{p q \perp}\right| g_{j}}{2}\right)=\int_{g_{j}^{2} \sigma^{2}}^{\infty} \mathrm{d} x x^{(-1 / 2)-n} \exp \left(-\frac{c_{p q \perp}^{2} g_{j}^{2}}{4 x}-x\right),
$$

can be evaluated recursively in terms of error functions.

The expression for the direct sum terms $D_{2 \mathrm{a}, p q}^{L}$ is given by

$$
D_{2 \mathrm{a}, p q}^{L}=\frac{2 \pi(-1)^{\ell}}{\Gamma\left(\ell+\frac{3}{2}\right)} \sum_{\substack{\mathbf{t}_{n} \\\left(t_{n}=\mathbf{c}_{p q \neq 0)}\right.}} Y_{L}^{*}\left(\mathbf{c}_{p q} \widehat{-t_{n}}\right) \frac{\Gamma\left(\ell+\frac{1}{2},\left|\mathbf{c}_{p q}-t_{n}\right|^{2} / 4 \sigma^{2}\right)}{\left|\mathbf{c}_{p q}-t_{n}\right|^{\ell+1} .}
$$

For $D_{2 \mathrm{~b}, p q}^{L}$ and $c_{p q \perp} \neq 0$ one obtains

$$
\begin{aligned}
D_{2 \mathrm{~b}, p q}^{L}= & -\delta_{m 0} \operatorname{sign}\left(c_{p q \perp}\right) \frac{\pi[\pi(2 \ell+1)]^{1 / 2}}{A} \frac{\Gamma(\ell+1)}{4^{\ell} c_{p q \perp}^{\ell-1}} \\
& \times \sum_{\ell / 2 \leq n \leq \ell} \frac{(-1)^{n} 4^{n}}{\Gamma(\ell-n+1) \Gamma(2 n-\ell+1)} \Gamma\left(n-\frac{1}{2}, c_{p q \perp}^{2} / 4 \sigma^{2}\right),
\end{aligned}
$$

while for $c_{p q \perp}=0 D_{2 \mathrm{~b}, p q}^{L}$ is given by

$$
D_{2 \mathrm{~b}, p q}^{L}= \begin{cases}\delta_{m 0}(2 \pi / A)\left[(-1)^{n}(4 n+1)^{1 / 2} /\left(4^{n} \sigma^{2} n-1\right)\right] \\ \times \Gamma\left(n-\frac{1}{2}\right) / \Gamma\left(2 n+\frac{3}{2}\right), & \ell=2 n, \text { even } \\ 0, & \ell \text { odd } .\end{cases}
$$


Finally for $D_{3, p q}^{L}$ the expression

$$
D_{3, p q}^{L}=-\delta_{p q} \delta_{L,(0,0)} \frac{2}{\sigma}
$$

is found. The potential can eventually be written in the following compact form as

$$
\begin{aligned}
V_{p}^{\text {Inter }}(\mathbf{r}) & =\sum_{L} V_{p, L}^{\mathrm{Inter}}(r) Y_{L}(\hat{\mathbf{r}}) \\
& =\sum_{L} \bar{V}_{p, \ell m}^{\mathrm{Inter}}(r) Y_{L}(\hat{\mathbf{r}})+\mathcal{A}\left(\frac{4 \pi}{3}\right)^{1 / 2} r Y_{10}(\hat{\mathbf{r}})+\left(\mathcal{A} c_{p \perp}+\mathcal{B}\right)(4 \pi)^{1 / 2} Y_{00}(\hat{\mathbf{r}}),
\end{aligned}
$$

where for matters of similarity with the three-dimensional translationally invariant case the expansion coefficients $\bar{V}_{p, \ell m}^{\text {Inter }}(r)$ are regrouped such that

$$
\begin{aligned}
& \bar{V}_{p, \ell m}^{\mathrm{Inter}}(r) \\
& \quad=\frac{4 \pi}{\Gamma\left(\ell+\frac{3}{2}\right)}\left(\sum_{L^{\prime}} \frac{(-1)^{\ell^{\prime}} \Gamma\left(\ell+\ell^{\prime}+\frac{3}{2}\right)}{\Gamma\left(\ell^{\prime}+\frac{1}{2}\right)} C_{\ell m,\left(\ell+\ell^{\prime}\right)\left(m^{\prime}-m\right)}^{\ell^{\prime} m^{\prime}} \sum_{q} G_{p q}^{\ell+\ell^{\prime}, m^{\prime}-m} Q_{q}^{L^{\prime}}\right)^{*} r^{\ell},
\end{aligned}
$$

and the constants $\mathcal{A}$ and $\mathcal{B}$ are fixed by the boundary conditions appropriate to the system investigated, namely on whether a surface or an interface is considered [8].

\section{3. 'Near field' corrections}

The problem of 'near field' corrections arises from the fact that by solving the Poisson equation in terms of an expansion in terms of multipole moments [15] only, this expansion a priori is no longer valid for near cells. By definition a cell centred at position $\mathbf{R}^{\prime}$ is called a near cell of a cell centred at $\mathbf{R}$ if

$$
\left|\mathbf{R}-\mathbf{R}^{\prime}\right|<r_{\mathrm{BS}}+r_{\mathrm{BS}}^{\prime},
$$

where $r_{\mathrm{BS}}$ denotes the radius of the bounding sphere (BS).

A real-space method which yields the exact intercell potential of neighbouring or near cells consists of: (1) an evaluation of the contributions to the intercell potential arising from near cells via a coordinate transformation of their respective intracell potentials, and (2) a sum over all near cells, the expansion coefficients of which in an angular momentum series being evaluated by means of a numerical angular integration.

The intracell potential of a particular charge distribution inside a cell with respect to the coordinate system centred in the same cell is given by equation (11) where the expansion coefficients of the shape truncated charge density are defined in equation (12). For $\mathbf{r}$ lying outside the bounding sphere this potential can be written in terms of multipole moments $Q_{\mathbf{R}}^{L}$,

$$
V_{\mathbf{R}}^{\mathrm{Intra}}(\mathbf{r})=\sum_{L} \frac{4 \pi^{1 / 2}}{r^{\ell+1}}\left(Q_{\mathbf{R}}^{L}\right)^{*} Y_{L}(\hat{\mathbf{r}}), \quad r>r_{\mathrm{BS}} .
$$


The intracell potential of a cell $\Omega^{\prime}$ centred at position $\mathbf{R}^{\prime}$ contributes then to the intercell potential of a cell $\Omega$ centred at $\mathbf{R}$ as follows

$$
\begin{aligned}
V_{\mathbf{R}^{\prime}}^{\mathrm{nf}}(\mathbf{r})= & V_{\mathbf{R}^{\prime}}^{\mathrm{Inta}}\left(\mathbf{R}-\mathbf{R}^{\prime}+\mathbf{r}\right) \\
= & \sum_{L} V_{\mathbf{R}^{\prime}, L}^{\mathrm{nf}}(r) Y_{L}(\hat{\mathbf{r}}) \\
= & \sum_{L} \frac{8 \pi}{2 \ell+1}\left(\left|\mathbf{R}-\mathbf{R}^{\prime}+\mathbf{r}\right|^{\ell} \int_{\left|\mathbf{R}-\mathbf{R}^{\prime}+\mathbf{r}\right|}^{r_{\mathrm{BS}}} \mathrm{d} r^{\prime}\left(r^{\prime}\right)^{-\ell+1} \bar{\rho}_{\mathbf{R}^{\prime} L}\left(r^{\prime}\right)\right. \\
& \left.+\left|\mathbf{R}-\mathbf{R}^{\prime}+\mathbf{r}\right|^{-\ell-1} \int_{0}^{\left|\mathbf{R}-\mathbf{R}^{\prime}+\mathbf{r}\right|} \mathrm{d} r^{\prime}\left(r^{\prime}\right)^{\ell+2} \bar{\rho}_{\mathbf{R}^{\prime} L}\left(r^{\prime}\right)\right)^{*} Y_{L}\left(\mathbf{R}-\widehat{\mathbf{R}^{\prime}}+\mathbf{r}\right) \\
= & \sum_{L} V_{\mathbf{R}^{\prime}, L}^{\mathrm{Intra}}\left(\left|\mathbf{R}-\mathbf{R}^{\prime}+\mathbf{r}\right|\right) Y_{L}\left(\mathbf{R}-\widehat{\mathbf{R}^{\prime}}+\mathbf{r}\right),
\end{aligned}
$$

where the expansion coefficients can be obtained in terms of the following angular integral

$$
\begin{aligned}
V_{\mathbf{R R}^{\prime}, L}^{\mathrm{nf}}(r) & =\int_{\Omega} \mathrm{d} \hat{\mathbf{r}} V_{\mathbf{R R}^{\prime}}^{\mathrm{nf}}(\mathbf{r}) Y_{L}^{*}(\hat{\mathbf{r}}) \\
& =\int_{\Omega} \mathrm{d} \hat{\mathbf{r}} \sum_{L^{\prime}} V_{\mathbf{R}^{\prime} L^{\prime}}^{\mathrm{nntra}}\left(\left|\mathbf{R}-\mathbf{R}^{\prime}+\mathbf{r}\right|\right) Y_{L^{\prime}}\left(\mathbf{R}-\widehat{\mathbf{R}^{\prime}}+\mathbf{r}\right) Y_{L}^{*}(\hat{\mathbf{r}}) .
\end{aligned}
$$

By applying a rotation of the coordinate system such that the $z$ axis points in the direction of the respective near cell, the above two-dimensional angular integral can be reduced to a one-dimensional integral [9],

$$
\begin{aligned}
V_{\mathbf{R}^{\prime}, L}^{\mathrm{nf}}(r)= & \frac{2 \pi C_{\ell m}}{r\left|\mathbf{R}-\mathbf{R}^{\prime}\right|} \int_{\left|\mathbf{R}-\mathbf{R}^{\prime}\right|-r}^{\left|\mathbf{R}-\mathbf{R}^{\prime}\right|+r} \mathrm{~d} u\left[u P_{\ell}^{|m|}\left(\frac{r^{2}+\left|\mathbf{R}-\mathbf{R}^{\prime}\right|^{2}-u^{2}}{2 r\left|\mathbf{R}-\mathbf{R}^{\prime}\right|}\right)\right. \\
& \left.\times \sum_{\ell^{\prime}=|m|}^{\ell_{\max }^{\prime}} C_{\ell^{\prime} m} P_{\ell^{\prime}}^{|m|}\left(\frac{r^{2}-\left|\mathbf{R}-\mathbf{R}^{\prime}\right|^{2}-u^{2}}{2 u\left|\mathbf{R}-\mathbf{R}^{\prime}\right|}\right) V_{\mathbf{R}^{\prime}, \ell^{\prime} m}^{\text {Intra }}(u)\right],
\end{aligned}
$$

where $u=\left(\left|\mathbf{R}-\mathbf{R}^{\prime}\right|^{2}+r^{2}-2 r\left|\mathbf{R}-\mathbf{R}^{\prime}\right| s\right)^{1 / 2}>0, s=\cos \theta \in[-1,1]$. Clearly enough in a practical application the summation over $\ell^{\prime}$ can only be performed up to a certain $\ell_{\max }^{\prime}$.

Then by summing up the contributions from all near cells an expression for the near field corrections is found,

$$
V_{\mathbf{R}, L}^{\mathrm{nf}}(r)=\sum_{\mathbf{R}^{\prime} \in \mathrm{nc}} V_{\mathbf{R R}^{\prime \prime}, L}^{\mathrm{nf}}(r)
$$

In figure 1 the geometrical arrangement for this procedure is illustrated: one can see that the corrections arise only outside a sphere of radius $r_{\text {cor }}=\left|\mathbf{R}-\mathbf{R}^{\prime}\right|-r_{\mathrm{BS}}^{\prime}$. For a less symmetric neighbourhood, however, one has to define

$$
r_{\text {cor }}=\min _{\mathbf{R}^{\prime}}\left(\left|\mathbf{R}-\mathbf{R}^{\prime}\right|-r_{\mathrm{BS}}^{\prime}\right) .
$$




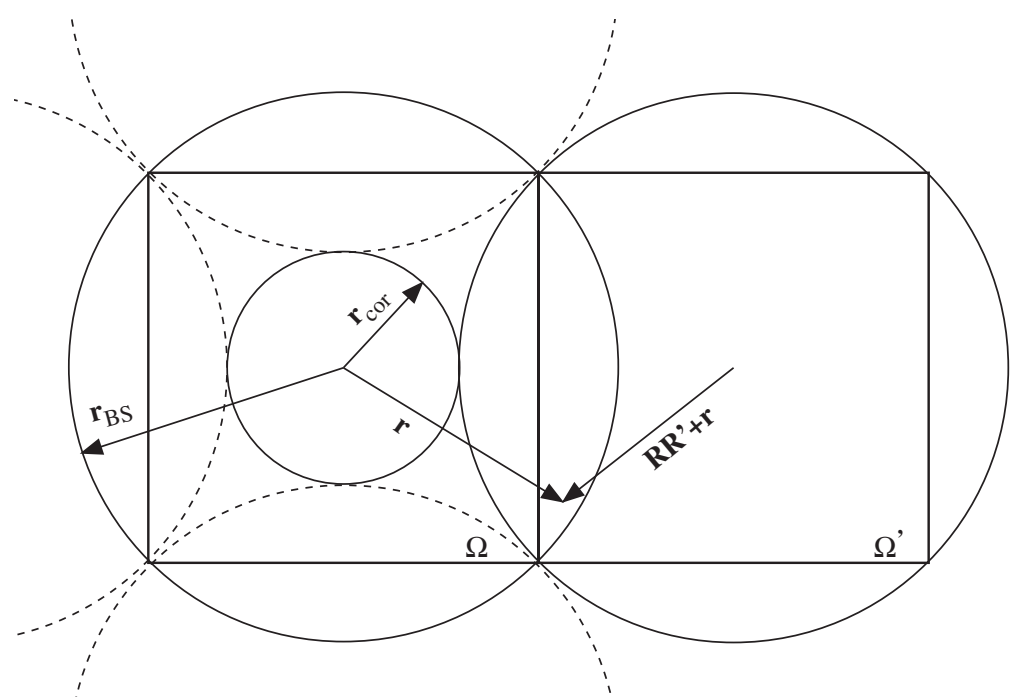

Figure 1. Illustration of two near cells with overlapping bounding spheres. Dashed circles denote bounding spheres of other near cells. Also indicated is the radius rcor outside which the near figeld corrections have to be calculated.

Consequently, the quantities $V_{L}^{\mathrm{nf}}(r)$ have to be calculated only for points $r>r_{\text {cor }}$ (and inside a sphere of radius $r_{\mathrm{BS}}$ ).

\subsection{Corrections to the intercell potential}

The correct intercell potential $\tilde{V}^{\text {Inter }}$ finally results from adding the near field correction term $V^{\mathrm{nf}}$ and subtracting the (incorrect) contributions from the near cells $V^{\mathrm{nc}}$, implicitly contained in the previously derived Madelung potential $V^{\mathrm{Inter}}$,

$$
\tilde{V}_{\mathbf{R}}^{\text {Inter }}(\mathbf{r})=V_{\mathbf{R}}^{\text {Inter }}(\mathbf{r})+\left(V_{\mathbf{R}}^{\mathrm{nf}}(\mathbf{r})-V_{\mathbf{R}}^{\mathrm{nc}}(\mathbf{r})\right),
$$

where the differences $V_{\mathbf{R}}^{\mathrm{nf}}(\mathbf{r})-V_{\mathbf{R}}^{\mathrm{nc}}(\mathbf{r})$ are the actual near field corrections to the intercell potential. In order to calculate $V_{\mathbf{R}}^{\text {nc }}(\mathbf{r})$ the following real-space sum has to be evaluated

$$
V_{\mathbf{R}}^{\mathrm{nc}}(\mathbf{r})=2 \sum_{\mathbf{R}^{\prime} \in \mathrm{nc}(\mathbf{R})} \sum_{L} \frac{(4 \pi)^{1 / 2}}{2 \ell+1}\left(\sum_{L^{\prime}} A_{\mathbf{R}^{\prime}}^{L L^{\prime}} Q_{\mathbf{R}^{\prime}}^{L^{\prime}}\right)^{*} r^{\ell} Y_{L}(\widehat{\mathbf{r}}),
$$

where the real space summation includes all near cells and the $A_{\mathbf{R R}_{n}}^{L L^{\prime}}$ are the corresponding real space Madelung constants (21). Figure 2 illustrates the angular momentum components of the potential arising from the near field corrections defined in equation (58) and the near cell potential in equation (62) for the case of fcc bulk Ag. In particular, the bottom of this figure demonstrates that the corrections indeed vanish for $r<r_{\text {cor }}$ and that all angular momentum components are rather small up to the muffin-tin radius. Beyond the muffin-tin region the 

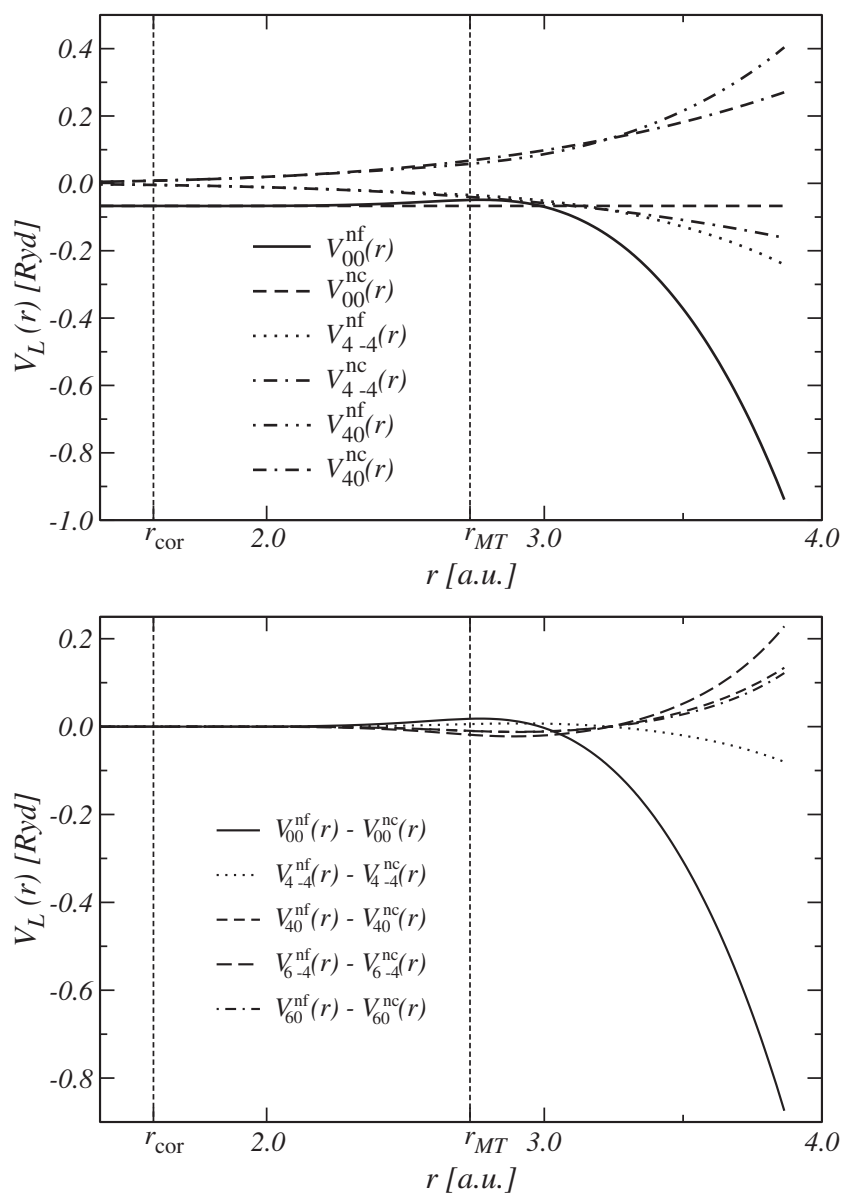

Figure 2. Top: the first three non-vanishing angular momentum components of the near field potential $V_{\ell m}^{\mathrm{nf}}(r)$ and the near cell potential $V_{\ell m}^{\mathrm{nc}}(r)$ for fcc bulk Ag. The dashed vertical lines indicate the muffin-tin radius $r_{\mathrm{MT}}$ and $r_{\text {cor }}$, respectively. Note the different behaviour of the spherically symmetric components between $r_{\mathrm{MT}}$ and $r_{\mathrm{BS}}$ : inside the muffin tin the differences are quite small, inside a sphere of radius $r_{\text {cor }}$ the components are identical. Bottom: angular momentum components of the near field corrections $V_{\ell m}^{\mathrm{nf}}(r)-V_{\ell m}^{\mathrm{nc}}(r)$ for fcc bulk Ag. For $r<r_{\text {cor }}$ the corrections are zero.

spherical symmetric component picks up the character of the intracell potential of a near cell. This feature is also reflected in figure 3 where the near field corrected intercell potential (cf. equation (61)) and the near field corrections are plotted along two directions in the Wigner-Seitz cell. Along the nearest neighbour direction the corrections are small up to the muffin-tin radius and then diverge in the same manner as the intracell potential of the neighbouring cell. Applying shape functions this behaviour is suppressed as they force the potential to jump to zero at the cell boundary. Along the direction of the farthest corner of the cell the corrections are small as compared with the intercell potential up to the bounding sphere radius. 


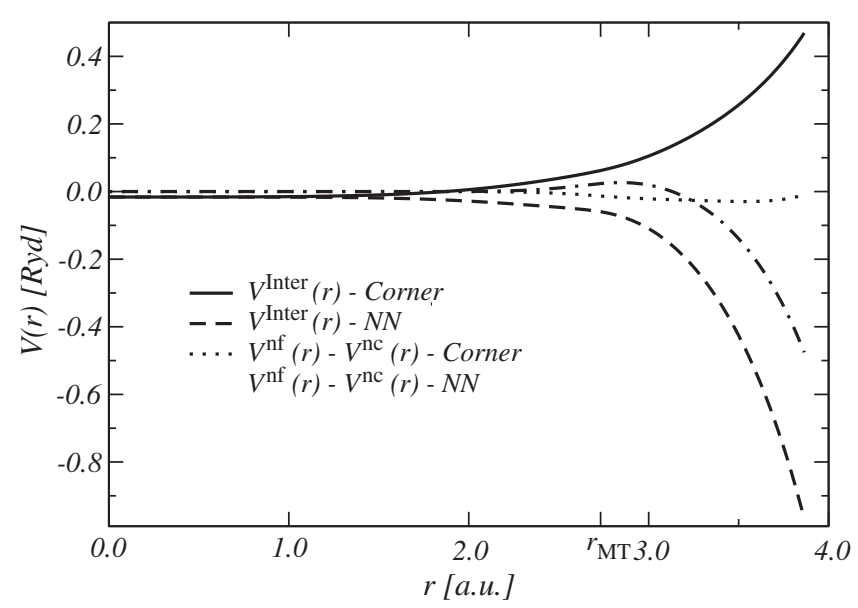

Figure 3. Intercell potential including the near field corrections plotted along two different directions of a fcc Wigner-Seitz cell for the case of bulk Ag. The solid line illustrates the potential in direction of the farthest corner (Corner) and the dashed line in direction of a nearest neighbour $(\mathrm{NN})$. Also shown are the near field corrections $V^{\mathrm{nf}}(\mathbf{r})-V^{\mathrm{nc}}(\mathbf{r})$ along the same directions.

\section{Numerical tests}

In order to test the accuracy of the method described above we have calculated the electrostatic potential and the electrostatic energy of two different charge distributions, the analytical and the numerical solutions of which can be directly compared.

As a first test case we considered a homogeneous electron density in a background of a positively charged point lattice, i.e. a system, which is also known as jellium or 'Slater-de Cicco' test [5].

In our second test a slowly varying, periodic charge density (without point charge singularities) reflecting the predominant character of the charge distributions in the interstitial regions was used. Such a density was first discussed by Morgan [16] and is indeed very useful to test the accuracy of the non-spherical contributions to the potential. Based on these two well-defined models we want to illustrate the actual effect of the NFC and to estimate the convergence with respect to the angular momentum summations. We compare our results to the ones of the recently proposed removed sphere method (RSM) [6], which provides an alternative to the present approach.

\subsection{Slater-de Cicco (jellium) test}

In this case the distribution of positive point charges is given by

$$
\rho_{+}(\mathbf{r})=Z \sum_{n} \delta\left(\mathbf{r}-\mathbf{t}_{n}\right)
$$


where $Z$ is the core charge and $\mathbf{t}_{n}$ are real space lattice vectors. The electron density is constant,

$$
\rho_{-}(\mathbf{r})=-\rho_{0}, \quad \rho_{0}=\frac{Z}{V},
$$

$V$ being the volume of the unit cell. Consequently, the angular momentum components of the truncated charge density are given by

$$
\bar{\rho}_{L}(r)= \begin{cases}\rho_{0} \sigma_{(0,0)}(r)-\delta(r) Z / r^{2}, & L=(0,0), \\ \rho_{0} \sigma_{L}(r), & L \neq(0,0) .\end{cases}
$$

For our test we used a lattice constant of 1 a.u. and a net cell charge of $Z=1$.

The analytic solution of Poisson's equation (4) can be obtained by means of Ewald's method and is given by

$$
\begin{aligned}
V(\mathbf{r})= & 2 Z\left(\sum_{\mathbf{t}_{n}} \frac{1}{\left|\mathbf{r}-\mathbf{t}_{n}\right|} \operatorname{erfc}\left(\frac{\left|\mathbf{r}-\mathbf{t}_{n}\right|}{2 \sigma}\right)+\frac{4 \pi}{V} \sum_{\substack{\mathbf{g}_{j} \\
\left(\mathbf{g}_{j} \neq 0\right)}} \frac{\exp \left(-\sigma^{2} \mathbf{g}_{j}^{2}\right)}{\mathbf{g}_{j}^{2}} \exp \left(\mathbf{i g}_{j} \cdot \mathbf{r}\right)\right) \\
& -8 \pi \rho_{0} \sigma^{2}+V_{0},
\end{aligned}
$$

where $\sigma$ is the Ewald parameter and $V_{0}$ is an arbitrary constant, which is unimportant since only differences of potentials and energies will be considered.

In figure 4 the difference between the potential in equation (66) and the numerically calculated electrostatic potential is plotted along different directions inside a fcc Wigner-Seitz cell. We used $\ell_{\max }=2,3$ and 4 in the multiple scattering expansions,

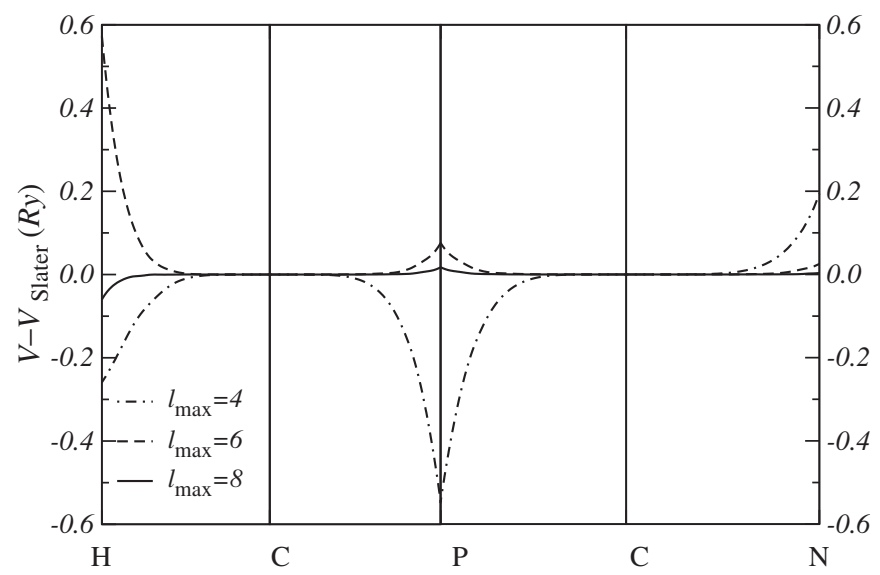

Figure 4. Difference between the numerically and analytically calculated potentials in the 'Slater-de Cicco' test as plotted along the major directions inside a fcc cell. The labelling of points are as follows: ' $\mathrm{P}$ ' is a near, ' $\mathrm{H}$ ' a far corner, ' $\mathrm{N}$ ' the centre of a plane and ' $\Gamma$ ' the cell centre. Different line styles refer to different numbers of angular moments used for the potential and the charge density: dash-dotted line: $\ell_{\max }=4$, dashed line: $\ell_{\max }=6$, solid line: $\ell_{\max }=8$. 
implying that the charge density and the potential were expanded up to $\ell_{\max }=4,6$ and 8 . This is the case for the 'outer' angular momentum summation, however, there also have to be 'inner' summations performed, as can be seen from equations (13) and (23) for the intra- and intercell potential, and in equation (17) for the multipole moments. In principle, by evaluating an angular momentum expansion of the Green's function up to $\ell_{\max }$, these inner sums can be extended up to $2 \ell_{\max }$. However, it is also possible to take better account of the shape of the truncated charge density by including higher components of the shape functions, and this in fact also leads to a convergence of the potential as will be discussed in section 4.2. Investigating equation (17) one finds that if $L^{\prime}$ is extended to $2 \ell_{\max }$ and $L^{\prime \prime}$ to $8 \ell_{\max }$, then one gets multipole moments up to $6 \ell_{\max }$. We find this procedure quite reasonable since the untruncated charge density converges more rapidly than the truncated charge density. In the latter the essential contributions are due to the shape of the cell and hence it is quite desirable to include more components of the shape functions. Similarly, the intracell potential can be evaluated for higher moments and can (together with the multipoles) be used in the calculation of the near field corrections in equation (58). Furthermore, the multipole moments can be used to improve the accuracy of equation (23), where, in addition, only higher terms of the Madelung constants need to be calculated.

The electrostatic (Coulomb) energy follows from the expression,

$$
U=\frac{1}{2} \int_{\Omega} \mathrm{d} \mathbf{r} \rho(\mathbf{r}) V(\mathbf{r})=\frac{1}{2} \int_{\Omega} \mathrm{d} \mathbf{r}\left(\rho_{+}(\mathbf{r})+\rho_{-}(\mathbf{r})\right) V(\mathbf{r}) .
$$

Inserting the charge densities in equations (63) and (64) and the potential in equation (66) one gets,

$$
\begin{aligned}
U= & -\left(\frac{Z^{2}}{r_{\mathrm{ASA}}}\right) \frac{r_{\mathrm{ASA}}}{a}\left(\frac{4 \pi \sigma^{2}}{V}+\frac{1}{\pi^{1 / 2} \sigma}-\frac{1}{2 \sigma} \sum_{\substack{\mathbf{t}_{n} \\
\left(\mathbf{t}_{n} \neq 0\right)}} \frac{2 \sigma}{\left|\mathbf{t}_{n}\right|} \operatorname{erfc}\left(\frac{\left|\mathbf{t}_{n}\right|}{2 \sigma}\right)\right. \\
& \left.-\frac{4 \pi \sigma^{2}}{V} \sum_{\substack{\mathbf{g}_{j} \\
\left(\mathbf{g}_{j} \neq 0\right)}} \frac{\exp \left(-\sigma^{2} \mathbf{g}_{j}^{2}\right)}{\sigma^{2} \mathbf{g}_{j}^{2}}\right),
\end{aligned}
$$

with $r_{\mathrm{ASA}}=(3 V / 4 \pi)^{1 / 3}$, i.e. an expression that can be calculated as reference value for different crystal structures.

For fcc and bcc lattices, figure 5 shows the convergence of the numerically calculated Coulomb energy as a function of $\ell_{\max }$ as compared to the exact value given by equation (68). If the NFCs are neglected and the inner sums are carried out only up to $2 \ell_{\max }$ the energy tends to a value which differs by about $10^{-3}$ (fcc) and $0.5 \times 10^{-3}$ (bcc) from the exact one. By simply extending the inner angular momentum sums to $6 \ell_{\max }$ (but still not including NFCs) the energies now match up to about $10^{-5}$. The reason for this good correlation will be considered in the next section by discussing the other type of test case. Clearly by including NFCs the converged values are in even better agreement (about $10^{-6}$ for both fcc and bcc) with the exact ones. 

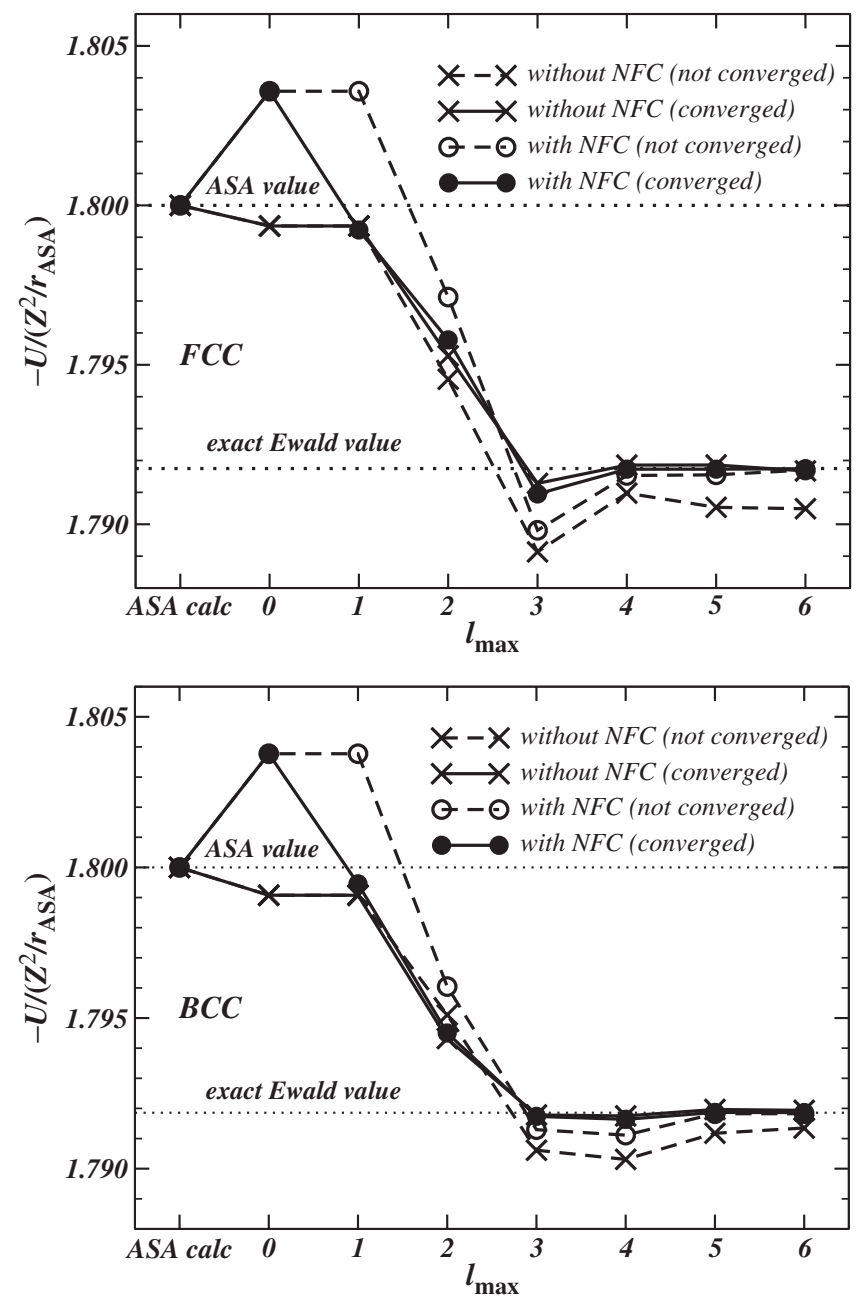

Figure 5. Angular momentum convergence of the electrostatic energy derived from a constant electron charge density for a fcc (top) and bcc (bottom) lattice. Circles and crosses depict results with and without near field corrections (NFC), respectively. Dashed lines indicate that the inner sums are extended up to $2 \ell_{\max }$ (not converged), solid lines refer to inner sums up to $6 \ell_{\max }$ (converged).

\subsection{Morgan test}

Consider the following charge density,

$$
\rho(\mathbf{r})=B \sum_{\mathbf{g}_{j} \in \mathrm{NN}^{r}(\mathbf{0})} \mathrm{e}^{\mathrm{i} \mathbf{g}_{j} \cdot \mathbf{r}},
$$

with $B$ being an arbitrary constant. Since the summation runs over the first reciprocal lattice vector shell $\mathrm{NN}^{r}(\mathbf{0})$, this charge distribution has the full 
symmetry of the lattice under consideration. By making use of Bauer's formula [15] one can find an analytic expression for the expansion coefficients of the charge density,

$$
\rho_{L}(r)=4 \pi B \mathbf{i}^{\ell} j_{\ell}(|\mathbf{g}| r) \sum_{\mathbf{g}_{j} \in \mathrm{NN}^{r}(\mathbf{0})} Y_{L}\left(\hat{\mathbf{g}}_{j}\right)
$$

The total potential due to such a charge distribution is given by

$$
V(\mathbf{r})=\frac{8 \pi \rho(\mathbf{r})}{\mathbf{g}^{2}}+V_{0},
$$

and its angular momentum components as

$$
V_{L}(r)=32 \pi^{2} B \mathrm{i}^{\ell} \frac{j_{\ell}(|\mathbf{g}| r)}{\mathbf{g}^{2}} \sum_{\mathbf{g}_{j} \in \mathrm{NN}^{r}(0)} Y_{L}^{*}\left(\hat{\mathbf{g}}_{j}\right)+(4 \pi)^{1 / 2} V_{0} \delta_{L, 00} .
$$

For our tests we chose $B=1$ and the lattice constant $a=1$ bohr.

Figure 6 illustrates the difference of the calculated electrostatic and the exact Morgan potential. As in the case of the jellium model (note, however, the different scales) the quality of agreement between the curves depends on the direction inside the Wigner-Seitz cell and on $\ell_{\max }$.

In figure 7 the convergence of the potential in terms of the internal angular momentum summation is illustrated. The potential in this case was calculated up to $\ell_{\max }=6$ and the internal angular momentum summation varied up to $\ell_{\max }=18$. For this value a reasonable convergence of the potential was achieved. Neglecting near field corrections and only extending the interior angular momentum summation of the intra- and intercell potential leads to an improved agreement of the potentials over a wider range of radial mesh points but is still substantially wrong for large radii.

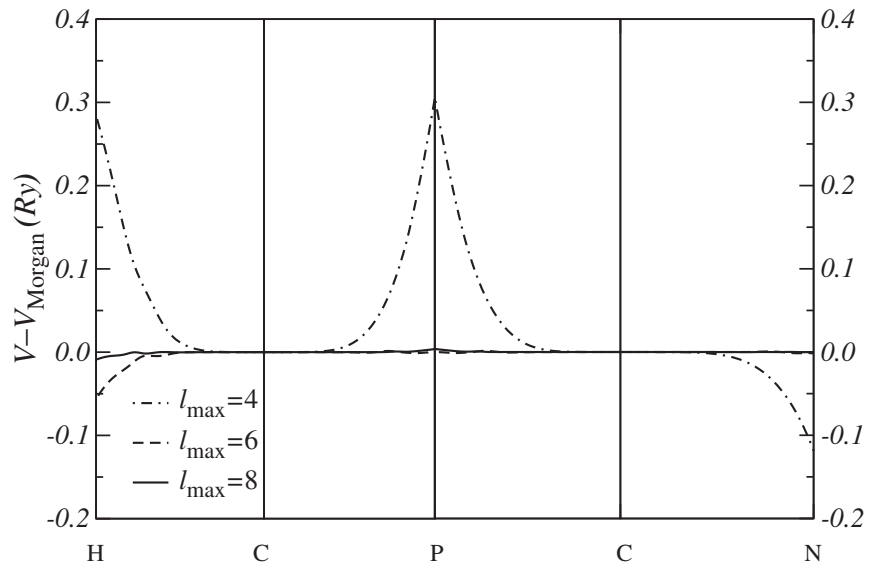

Figure 6. Difference between the numerically calculated and the exact potentials of the Morgan test along the major directions inside a fcc Wigner-Seitz cell. The labelling of points is as follows: ' $\mathrm{P}$ ' is a near, ' $\mathrm{H}$ ' a far corner, ' $\mathrm{N}$ ' the centre of a plane and ' $\Gamma$ ' the cell centre. Different line styles refer to different numbers of angular moments used for the potential and the charge density: dash-dotted: $\ell_{\max }=4$, dashed line: $\ell_{\max }=6$, solid line: $\ell_{\max }=8$. 


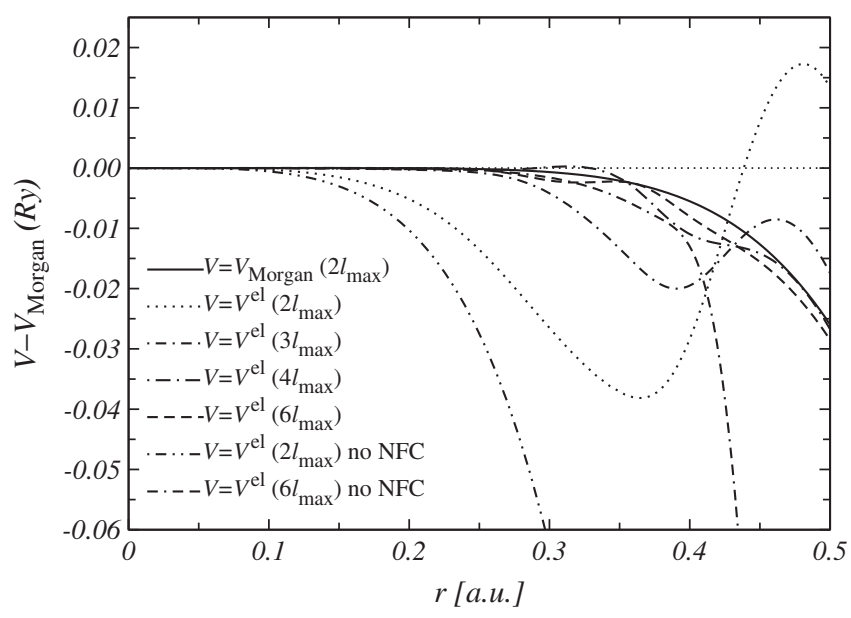

Figure 7. Convergence of the numerically calculated potential of the Morgan test in terms of the internal angular momentum summation $\left(V^{\mathrm{el}}=V^{\mathrm{Inter}}+V^{\mathrm{Intra}}\right)$. For the calculations of the Green's function $\ell_{\max }=3$ has been used. Shown are the differences between the analytical potential and the numerically calculated electrostatic potential in the direction from the cell centre to a far corner $(\Gamma \mathrm{H})$ in a fcc Wigner-Seitz cell, where the best possible match is displayed by the solid line. This curve is obtained when the expansion coefficients of the Morgan potential are calculated by means of equation (72) and then summed up to $2 \ell_{\max }=6$. All other curves refer to the numerically calculated potential where the 'outer' summation also extends to $2 \ell_{\max }=6$, but the 'inner' angular momentum summation is varied between $2 \ell_{\max }=6$ and $6 \ell_{\max }=18$. Also shown are curves where the NFC have not been included in the calculation. One can see that simply by extending the inner summation up to $6 \ell_{\max }$ the results are considerably improved.

The electrostatic energy is given by

$$
U=\frac{4 \pi}{\mathbf{g}^{2}} B^{2} V \sum_{\mathbf{g}_{j} \in \mathrm{NN}^{r}(\mathbf{0})} 1,
$$

and its convergence with respect to $\ell_{\max }$ is shown in figure 8 . For a fcc cell the exact value is $2 a^{5} / 3 \pi$ and for a bcc cell $3 a^{5} / \pi$ if $a$ is the lattice constant which has been set to unity in our test. If the NFCs are not included in the calculation the energy does not converge to the correct values as is evident from figure 8 . The deviation is of the order of $10 \mathrm{mRy}$. Inclusion of the NFCs then results in a quite rapid convergence: for $\ell_{\max }=3$ the exact energy is obtained within $2 \mathrm{mRy}$. As in the Slater-de Cicco test, without NFCs but by converging the internal angular momentum sums a good convergence of the energy is observed. Even though there is a considerable aberration from the exact potential, the electrostatic energy yields almost the exact value. This is due to the fact that the difference $V(\mathbf{r})-V_{\text {Morgan }}(\mathbf{r})$ tends to oscillate around zero for a given radius as a function of the angles, resulting thus in contributions which mostly cancel in the necessary integration over the Wigner-Seitz cell when calculating the energy. We have illustrated this in figure 9 where the differences to the exact Morgan potential are plotted for a plane through the cell centre. One can see not only the oscillations 

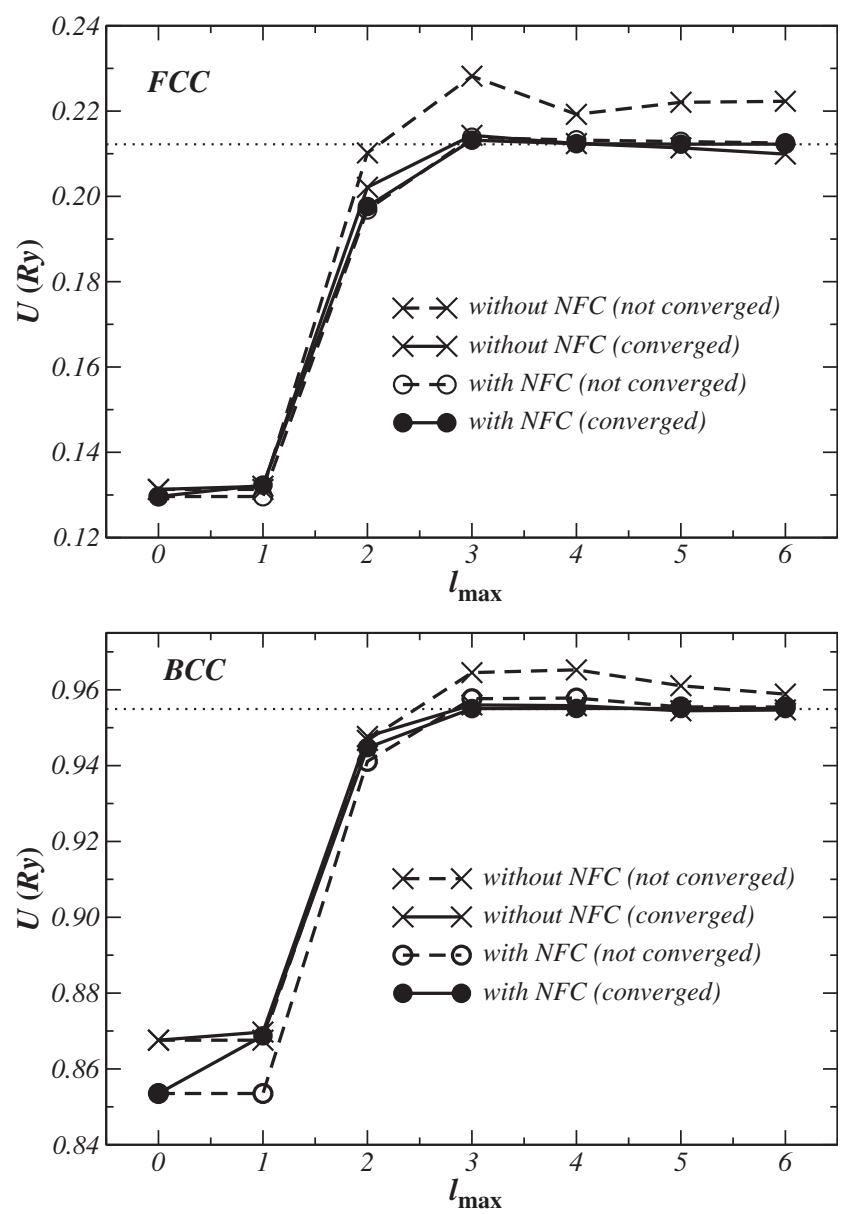

Figure 8. Angular momentum convergence of the electrostatic energy for a fcc (top) and bcc (bottom) lattice in the Morgan test. Circles depict calculations including NFCs and crosses those without NFCs.

around zero but also that with increasing angular momentum the aberrations are pushed further outwards and become smaller in size thus giving smaller contributions to the integrals.

\subsection{Comparison with RSM method}

The recently proposed so-called removed sphere method (RSM) [6] is conceptually simple and numerically very efficient. It is based on the fact that the electrostatic potential can also be written in the form

$$
V(\mathbf{r})=\sum_{L}\left(V_{L}^{\mathrm{e}}(r)+a_{L} r^{\ell}\right) Y_{L}(\hat{\mathbf{r}}), \quad r<r_{\mathrm{BS}},
$$

where $V_{L}^{\mathrm{e}}(r)$ is obtained from equation (11) but with the untruncated instead of the truncated charge density. The coefficients $a_{L}$ are unknown and need to be 
(a)

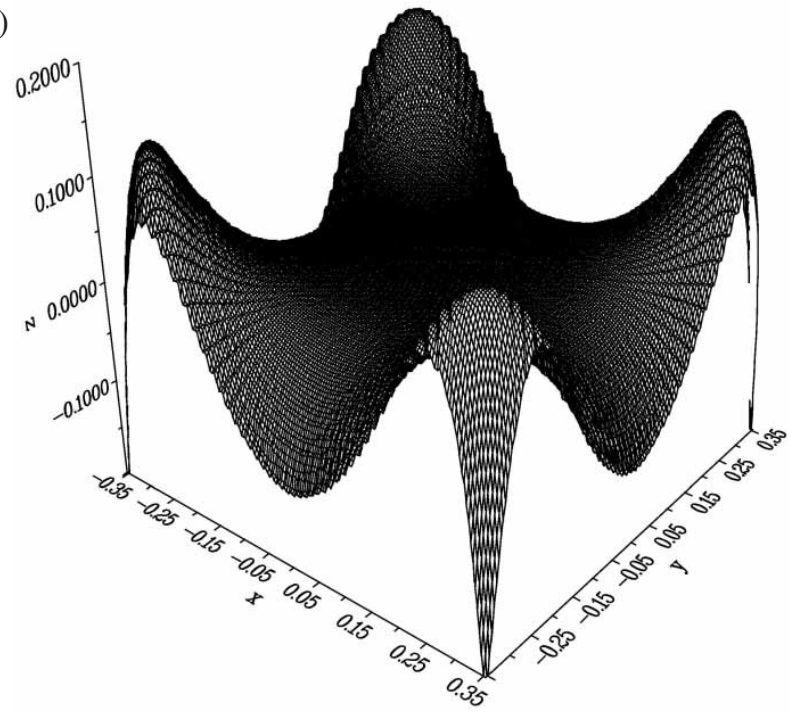

(b)

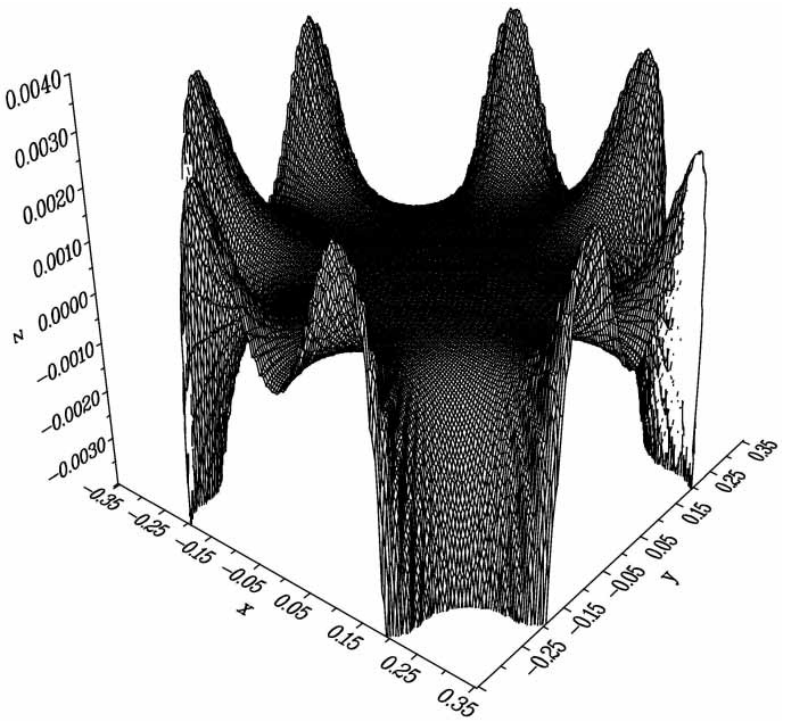

Figure 9. Difference of the numerical and exact Morgan potentials (in Rydberg) for $\ell_{\max }=2$ (top) and $\ell_{\max }=3$ (bottom) where the internal sums have been extended up to $6 \ell_{\max }$ and the NFC were not included in the calculation. The plots show the values inside a Wigner-Seitz cell for a (100) plane through the cell centre where $x$ and $y$ are in units of bohr.

calculated. Since for small $r<r_{\text {cor }}$ the potential is also given by the sum of intracell and uncorrected intercell contribution

$$
V(\mathbf{r})=\sum_{L}\left(V_{L}^{\mathrm{Intra}}(r)+V_{L}^{\mathrm{Inter}}(r)\right) Y_{L}(\hat{\mathbf{r}}), \quad r<r_{\mathrm{cor}}
$$


(a)
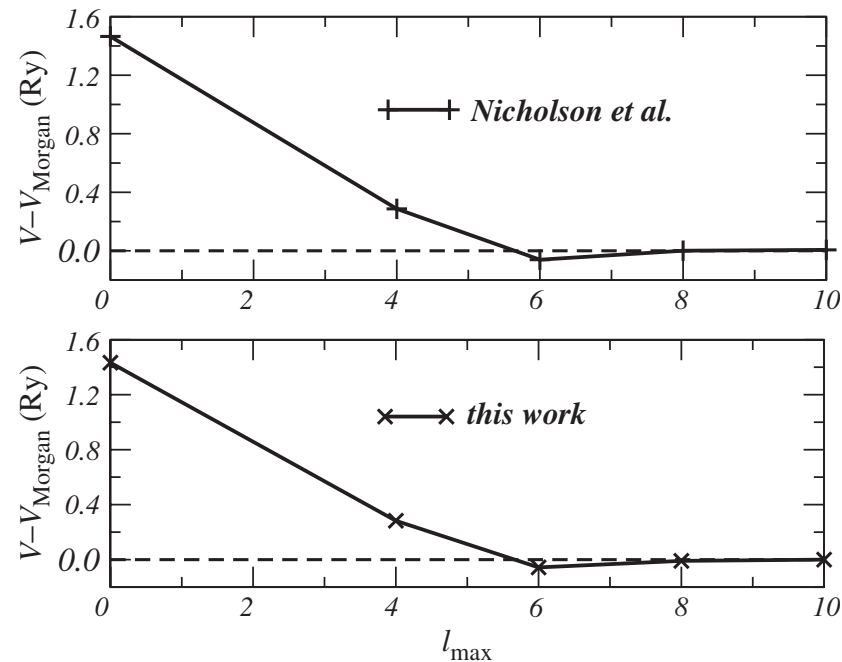

(b)
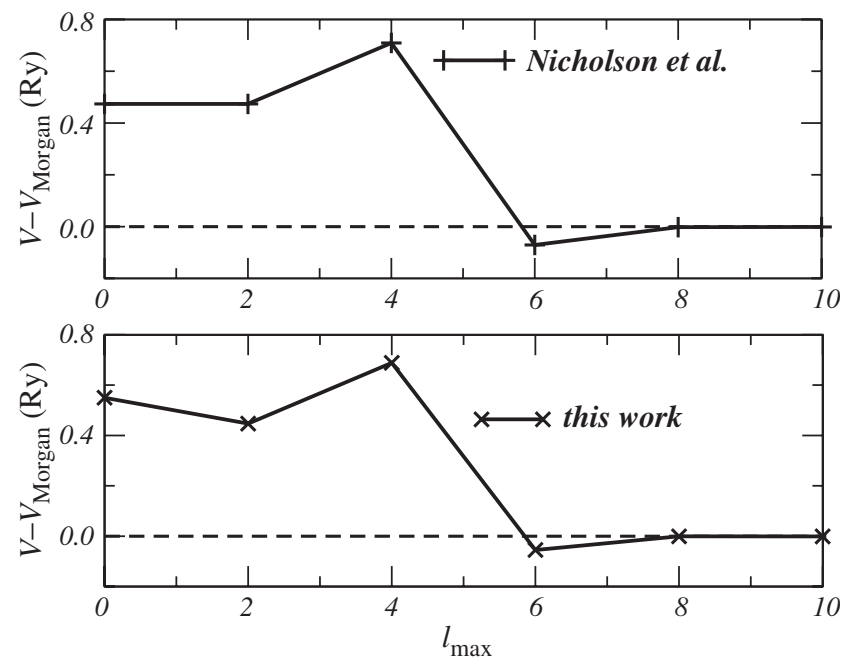

Figure 10. Difference of the calculated and the exact potential due to the Morgan charge density (69) as a function of $\ell_{\max }$ at the farthest corner of a (a) fcc, and (b) bcc Wigner-Seitz cell. A comparison between the values obtained with the method described in this work and that of Nicholson et al. [6] is displayed.

the coefficients $a_{L}$ can be calculated by setting (74) equal to (75) for small $r$. This leads to potentials which are essentially equal compared to the ones obtained with the method presented here up to $\ell \leq 10$ (cf. figure 10). If one uses up to even higher $\ell_{\max }$ (and the characteristic shape functions of the cells), however, numerical convergence problems can arise. Due to the shape functions there are non-vanishing high- $L^{\prime}$ multipole moments present (even for constant charge densities) which lead for near cells and high $L$ to slowly converging $L^{\prime}$ sums of the form $\sum_{L^{\prime}} A_{\mathbf{R}^{\prime}}^{L L^{\prime}} Q_{\mathbf{R}^{\prime}}^{L^{\prime}}$.

In our method this problem does not arise, because the multipole contributions from near cells in (23) and (62) cancel exactly. This problem might not occur 
if smoother but overlapping shape functions are used [17]. However, this type of shape function does not fit to the concept of non-overlapping potentials in multiple scattering theory [2].

\section{Conclusions}

We presented an effective tool for calculating the electrostatic potential from a given charge density which is especially suitable for multiple scattering methods and systems with two- or three-dimensional translational symmetry. In order to treat the contributions from near cells (the NFCs) correctly we have developed a physically intuitive method which relies on the idea that the intercell potential of one cell is the sum of the intracell potentials of all other cells.

Numerical tests show that the method is applicable and yields the correct results for two systems for which exact results can be obtained analytically. Our tests indicate that for methods which are based on angular momentum expansions it is very important to converge the 'inner' sums (or at least carry them further than the 'outer' ones). In particular it was shown that in order to calculate the potential it turns out to be essential to include near field corrections, whereas for the calculation of the total energy it is more important to converge the interior summations. The proposed method is in particular meant for semi-infinite systems and the numerical results shown for bulk systems serve as illustrations with respect to well-known, analytically solvable test problems.

\section{Acknowledgments}

We would like to thank Dr D.M.C. Nicholson for helpful discussions. Financial support of this work was provided by the Center for Computational Materials Science (Contract No. Zl. 98.366), the Research and Technological Cooperation Project between Austria and Hungary (Contract No. A-3/03) and the Hungarian National Scientific Research Foundation (OTKA T046267).

\section{References}

[1] L. Szunyogh, B. Újfalussy, P. Weinberger, et al., Phys. Rev. B 492721 (1994).

[2] J. Zabloudil, R. Hammerling, L. Szunyogh, et al., Electron Scattering in Solid Matter (Springer, Berlin, 2004).

[3] A. Gonis, E.C. Sowa and P.A. Sterne, Phys. Rev. Lett. 662207 (1991).

[4] L. Vitos and J. Kollár, Phys. Rev. B 514074 (1995).

[5] X.-G. Zhang, W.H. Butler, J.M. MacLaren, et al., Phys. Rev. B 4913383 (1994).

[6] D.M.C. Nicholson and W.A. Shelton, J. Phys. Condens. Matter 145601 (2002).

[7] K. Kambe, Z. Naturforschung 22a 322 (1967); Z. Naturforschung 22a 422 (1967); Z. Naturforschung 23a 1280 (1968).

[8] R. Hammerling, Aspects of dispersion interactions and of the full-potential Korringa-Kohn-Rostoker (KKR) method for semi-infinite systems. PhD thesis, Vienna University of Technology, Vienna (2003) (also at http://www.cms.tuwien.ac.at/ PhD_Theses). 
[9] Note that the complex spherical harmonics are defined as

$$
Y_{\ell m}(\theta, \varphi)=C_{\ell m} P_{\ell}^{|m|}(\cos \theta) \exp (\mathrm{i} m \varphi), \quad C_{\ell m}=\mathrm{i}^{m+|m|}\left(\frac{2 \ell+1}{4 \pi} \frac{(\ell-|m|) !}{(\ell+|m|) !}\right)^{1 / 2},
$$

with $P_{\ell}^{|m|}(\cos \theta)$ being the associated Legendre functions.

[10] Y. Wang, G.M. Stocks and J.S. Faulkner, Phys. Rev. B 495028 (1994).

[11] N. Stefanou, H. Akai and R. Zeller, Comp. Phys. Commun. 60231 (1990).

[12] N. Stefanou and R. Zeller, J. Phys. Condens. Matter 37599 (1991).

[13] I.S. Gradshteyn and I.M. Ryzhik, Table of Integrals, Series and Products, Corrected and Enlarged Edition (Academic Press Inc., New York, 1980).

[14] M. Abramowitz and I. Stegun, Handbook of Mathematical Functions (Dover Publ., New York, 1973).

[15] J.D. Jackson, Classical Electrodynamics (Wiley, New York, 1992).

[16] J. van W. Morgan, J. Phys. C: Solid State Phys. 101181 (1977).

[17] K. Koepernik and H. Eschrig, Phys. Rev. B 591743 (1999). 